\title{
A low-profile antenna with multi-directional beam pattern using loop elements
}

\author{
Nobuhiro Imaizumi ${ }^{\text {a) }}$ and Takeshi Fukusako ${ }^{\text {b) }}$ \\ Graduate School of Science and Technology, Kumamoto University, \\ Kurokami, Chuo-ku, Kumamoto 860-8555, Japan \\ a)imaizumi@st.cs.kumamoto-u.ac.jp \\ b) fukusako@cs.kumamoto-u.ac.jp
}

Abstract: A low-profile antenna having four directional beam patterns using loop elements has been proposed. The proposed antenna is vertically polarized and has four directional beams for sensor nodes. The thickness of the antenna is only $3.2 \mathrm{~mm}$ (0.11 effective wavelength) resulting in a lowprofile structure. Simulated and measured average gain of $8.0 \mathrm{dBi}$ and $5.6 \mathrm{dBi}$ are obtained, respectively.

Keywords: low-profile antenna, vertical polarization, multi-directional beam, wireless sensor network, sensor node

Classification: Antennas and Propagation

\section{References}

[1] S. Ishikawa and T. Ninomiya, "Polarization diversity antenna with L-shaped skit for wireless sensor networks," Asian Pacific Microwave Conference Proceeding (APMC), Kaohsiung, Taiwan, pp. 1181-1183, Dec. 2012. DOI:10. 1109/APMC.2012.6421863

[2] J.-P. Chen and P. Hsu, "A slot antenna with split-ring resonators for wireless sensor networks," 2013 IEEE Antennas and Propagation Society International Symposium (APSURSI), Orlando, Florida, USA, pp. 1794-1795, July 2013. DOI:10.1109/APS.2013.6711556

[3] M. Cardei, M. Thai, Y. Li, and W. Wu, "Energy-efficient target coverage in wireless sensor networks," Proceedings IEEE, INFOCOM 2005, 24th Annual Joint Conference of the IEEE Computer and Communications Societies, Miami, Florida, USA, pp. 1976-1984, Mar. 2005. DOI:10.1109/INFCOM. 2005.1498475

[4] S. Sharmin, F. N. Nur, M. A. Razzaque, M. M. Rahman, A. Almogren, and M. M. Hassan, "Tradeoff between sensing quality and network lifetime for heterogeneous target coverage using directional sensor nodes," IEEE Access, vol. 5, pp. 15490-15504, June 2017. DOI:10.1109/ACCESS.2017.2718548

[5] J. Liu, K. Xiong, P. Fan, and Z. Zhong, "RF energy harvesting wireless powered sensor networks for smart cities," IEEE Access, vol. 5, pp. 9348-9358, June 2017. DOI:10.1109/ACCESS.2017.2703847

[6] G. Giorgetti, A. Cidronali, S. K. S. Gupta, and G. Manes, "Exploiting low-cost directional antennas in $2.4 \mathrm{GHz}$ IEEE802.15.4 wireless sensor networks," European Conference on Wireless Technologies 2007, Munich, Germany, pp. 217-220, Oct. 2007. DOI:10.1109/ECWT.2007.4403985 
reconfigurable antenna for a wireless sensor network sink node," Asia-Pacific Microwave Conference 2010, Yokohama, Japan, pp. 2020-2024, Dec. 2010.

[8] T. Fujimoto, T. Tsuruoka, T. Fujishima, Y. Ishizuka, S. Sugimoto, and T. Sasamura, "Circularly polarized small microstrip antenna for wireless sensor networks," 2015 International Symposium on Antennas and Propagation (ISAP), Hobart, Tasmania, Australia, pp. 1-2, Nov. 2015.

[9] T. Fukusako and Y. Ogata, "A design of bi-directional and low-profile antenna using microstrip element," IEEE Antennas and Propagation in Wireless Communications (APWC2016), Cairns, Australia, pp. 89-90, Sept. 2016. DOI:10.1109/APWC.2016.7738126

[10] N. Imaizumi and T. Fukusako, "A low-profile surface wave antenna with bidirectional beam pattern using loop element," 2017 IEEE International Conference on Computational Electromagnetic (ICCEM), Kumamoto, Japan, pp. 226-227, Mar. 2017. DOI:10.1109/COMPEM.2017.7912761

\section{Introduction}

Wireless sensor networks have been recently used for such as industries, agriculture applications, medical and home networks. At the same time, applications of electromagnetics for sensing are getting attractive for wireless engineers and industrial society. Usually, the sensor nodes of wireless sensor networks include some integrated circuits, such as micro processor, A/D converter. Therefore, the antennas for sensor nodes require a small size and a simple structure. Several antennas have been recently proposed in $[1,2]$ for the above purposes for consideration of achieving simple designa and small sizes. However, we should note that most of the proposed antennas have omni-directional radiation patterns for now.

On the other hand, it is important to reduce total energy consumption for enlonging the lifetimes of network systems $[3,4,5]$ because some of them uses solar panels and batteries. As a technique for solving this purpose, directive antennas have been proposed $[6,7,8]$ in order to save the energy consumption for the system with the directional beam by focusing the radiated energy effectively on the required directions of sensors. However, there have been a new kind of problem in which the number of links are decreased by using directional antennas compare to omni-directional antennas. Therefore, it is important to have a number of directional beams. Moreover, the antennas of previous studies $[6,7,8]$ have complex structures and large sizes.

Considering these situations, we propose a directional antenna having a simple and low-profile structure in this letter. Originally, surface wave antennas have been known to have keen beams. Based on this background, some low-profile antennas radiating bi-directional beams by surface wave have been proposed by the authors' group [9, 10]. Low-profile structure can make antennas be inconspicuous in applications such as avoiding destroying scenery. Being developed from the previous structures, the proposed antenna has a low-profile structure and a radiation beam which is directed to four directions. This behaviors including mechanisms are discussed in the following sections. 


\section{Antennas structure}

Fig. 1 shows the top and side views of the proposed structure consisting of two loop elements, a feeding patch, two dielectric substrates and a ground plane. In this structure, total thickness $h$ is $3.2 \mathrm{~mm}\left(0.11 \lambda_{g}\right.$, where $\lambda_{g}$ is the effective wavelength), and the radius of the substrate is $34.5 \mathrm{~mm}$. In the proposed antenna, dielectric substrate is Taconic substrate that has a dielectric constant of $\epsilon_{r}=2.2$ and a loss tangent of 0.001 . The $8 \mathrm{~mm}$-square feeding patch is capacitively coupled with the two elements considering impedance matching. The feeding patch is installed between two dielectric substrates. A loop element has a dimension of $1 \mathrm{~mm}$ in width. A length of the long side $L$ is $66.79 \mathrm{~mm}\left(2.07 \lambda_{g}\right)$, and the length of short side $W$ is $11 \mathrm{~mm}\left(0.34 \lambda_{g}\right)$.

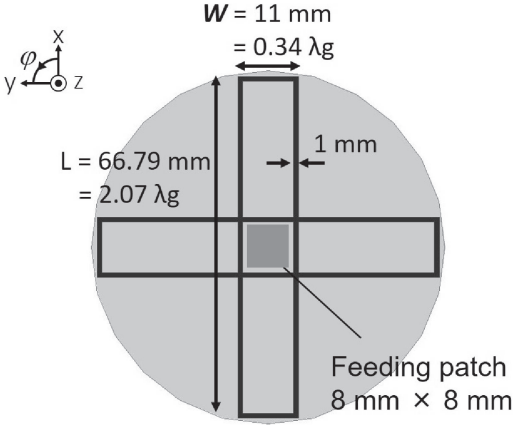

(a) Top View
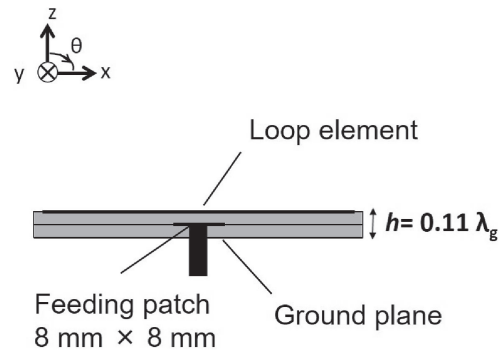

(b) Side View

Fig. 1. Antenna structure

\section{Antenna performances}

Fig. 2 shows the simulated and measured results of $\left|S_{11}\right|$ characteristics and radiation patterns. According to Fig. 2(a), the simulated resonant frequency is $6.28 \mathrm{GHz}$, and $-11.68 \mathrm{~dB}$ of $\left|S_{11}\right|$ has been obtained. On the other hand, the measured resonant frequency is $6.44 \mathrm{GHz}$, and measured $\left|S_{11}\right|$ at the resonant frequency is $-12.46 \mathrm{~dB}$. Comparing the simulated and measured resonant frequencies, we can find a discrepancy between them. This reason is probably due to an air gap between two dielectric substrates in the fabricated antenna.

Fig. 2(b) shows simulated and measured radiation patterns at the respective resonant frequencies. As seen from this figure, four beams are directed at $\phi=0^{\circ}$, $90^{\circ}, 180^{\circ}$ and $270^{\circ}$ in the $x y$-plane, and the simulated and measured average gains are about $8.1 \mathrm{dBi}$, and $5.6 \mathrm{dBi}$, respectively. The gain of the measured results is lower by $2.5 \mathrm{~dB}$ than the simulated results. Similarly, the descrepancy between the simulated and measured resonant frequencies is found. Furthermore, the directional beams are weakly radiated at also $\phi=45^{\circ}, 135^{\circ}, 225^{\circ}$ and $315^{\circ}$ in the $x y$-plane even though the radiation is attenuated in such directions according to the simulated result. The descrepancies in regard to the gain and the radiation pattern will be discussed in the next section. 


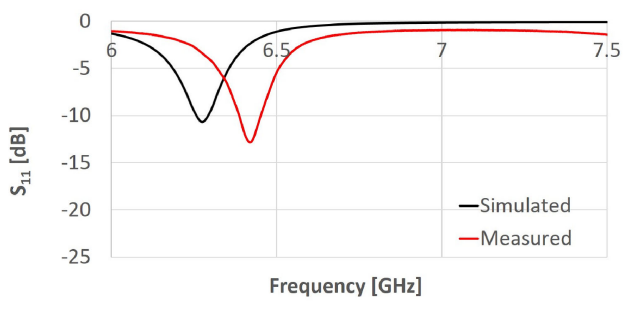

(a) $\mathrm{S}_{11}$ characteristics

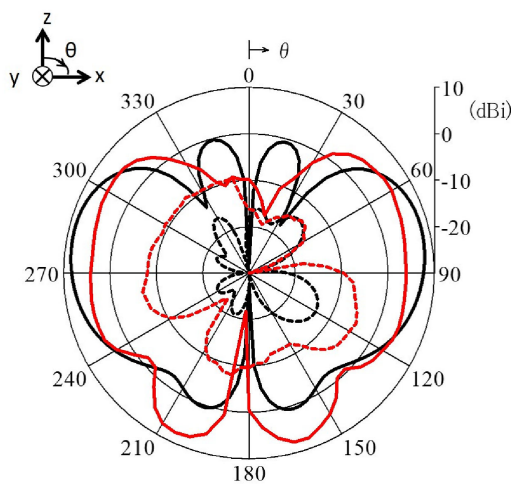

(c)Radiation pattern in $x z$-plane $\left(\phi=0^{\circ}\right)$

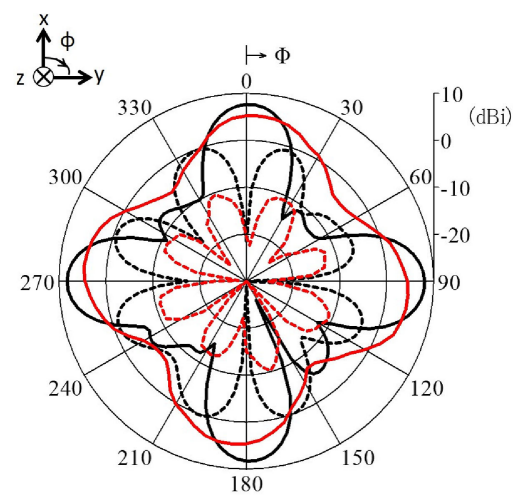

(b)Radiation pattern in $x y$-plane

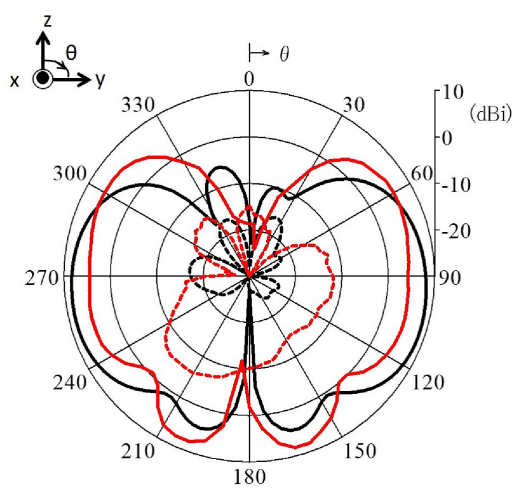

(d)Radiation pattern in $y z$-plane $\left(\phi=90^{\circ}\right)$

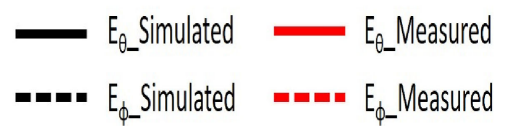

Fig. 2. Antenna characteristics

\section{Analysis}

As seen from Fig. 3(a), the electric fields show strong amplitude along the $x$-axis at four locations. Now, we labbel the four locations as A, B, C and D, respectively as shown in the figure. Fig. 3(b) also shows the distance in wavelength between each location. This shows that the distance between $\mathrm{A}$ and $\mathrm{B}$ is $0.87 \lambda_{g}, \mathrm{~A}$ and $\mathrm{C}$ is $1.18 \lambda_{g}$ and $\mathrm{A}$ and $\mathrm{D}$ is $2.04 \lambda_{g}$. Therefore, referencing the distances and considering the variation in phase with the locations including A-D in Fig. 3(c), we can understand that the electric fields at respective locations of A-D enhance each other as the radiations from the locations are almost in-phase. We can also have the same understanding along the $y$-axis. As a result, we can figure out that the four beams are directed in $\phi=0^{\circ}, 90^{\circ}, 180^{\circ}$ and $270^{\circ}$ in the $x y$-plane.

On the other hand, the electric fields shows strong amplitude along the line of the $\phi=45^{\circ}$ with respect to $x$-axis at four locations in the Fig. 3(d). We call the four locations i, ii, iii and iv, respectively. The Fig. 3(e) shows the distance between each location of i-iv. As seen from Fig. 3(f), the electric field at respective locations of $i-i v$ are in phase. Since the distance between $i$ and iii is $0.40 \lambda_{g}$ and that between $\mathrm{i}$ and iv is $0.44 \lambda_{g}$, we can understand that the electric fields at $\mathrm{i}-\mathrm{iv}$ 


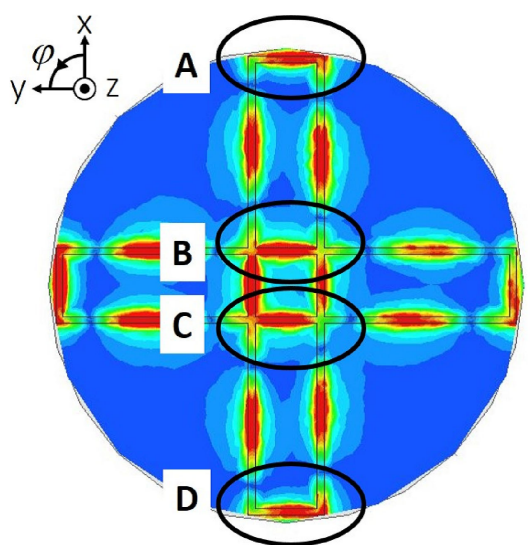

(a)Electric fields

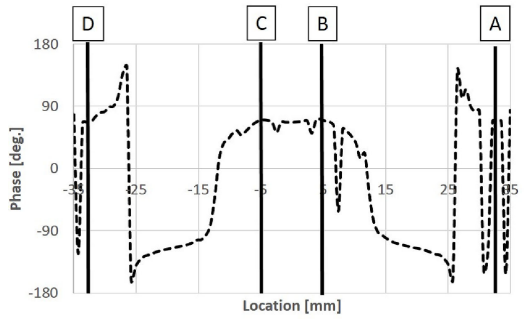

(c)Phase distribution $\left(\phi=0^{\circ}\right.$ to $\left.180^{\circ}\right)$

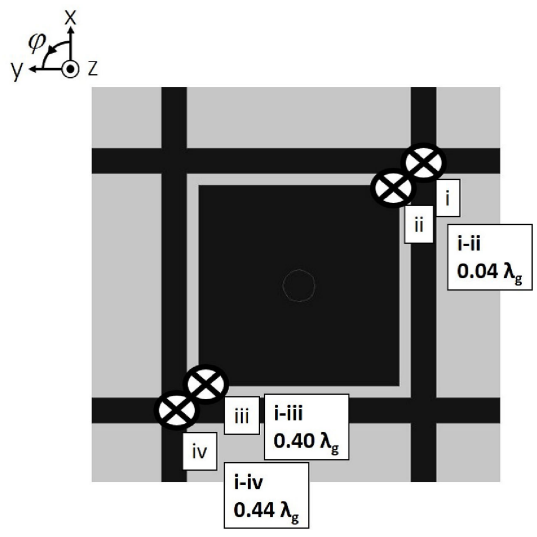

(e)Distance between labbeled locations

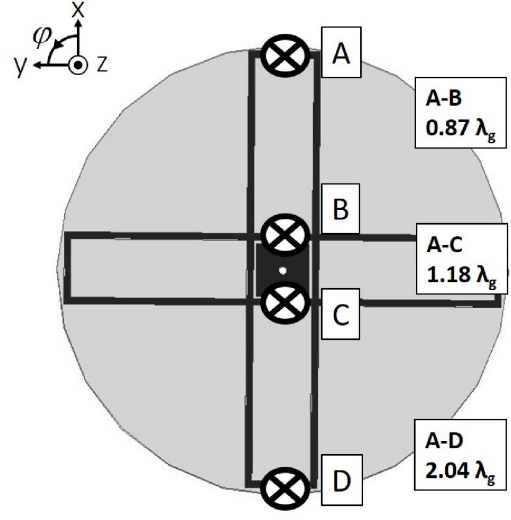

(b)Distance between labbled locations

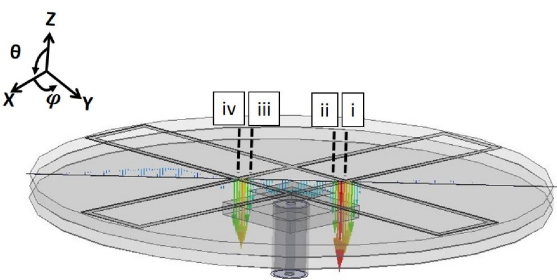

(d)Electric fields

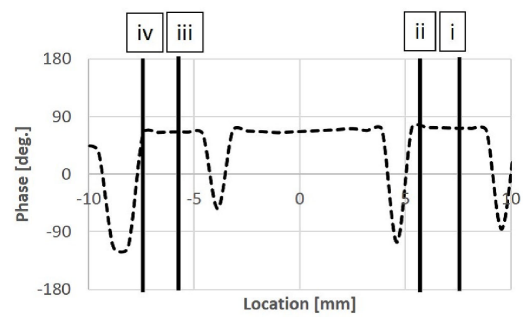

(f)Phase distribution $\left(\phi=45^{\circ}\right.$ to $\left.225^{\circ}\right)$

Fig. 3. Electric fields, distance between labbeled locations and phase distribution

cancel out each other considering that the interval between $\mathrm{i}-\mathrm{iv}$ are close to $\lambda_{g} / 2$. As a result, we can conclude that the directional beams are weakly radiated in $\phi=45^{\circ}, 135^{\circ}, 225^{\circ}$ and $315^{\circ}$ in the $x y$-plane. As discussed here, the diagonal length around the locations should be $\lambda_{g} / 2$ to cancel the radiation in these angles. In the present design, we consider that the current design around the structure in Fig. 3(e) is also involved in a good impedance matching.

\section{Conclusion}

In this letter, we have discussed a low-profile antenna using loop elements for the purpose of reducing the energy consumption and four links for the sensor nodes. Using planar elements, the proposed antenna has a low-profile structure and multi- 
directional beams by combining loop elements. The beams are directed at every $90^{\circ}$ in $x y$-plane so that high gain can be achieved even though the antenna dimension is relatively small. However, the cross-polarization yields energy loss. Decreasing the cross polarization is a future work. 